\title{
Proposal of Method to Detect Luggage Lifting
}

\author{
Yuhki Kitazono ${ }^{\text {a, }}$, Toru Tanaka ${ }^{\mathrm{a}}$, Takakazu Mouri ${ }^{\mathrm{a}}$, \\ Yuji Ohta ${ }^{\mathrm{b}}$, Shota Nakashima ${ }^{\mathrm{c}}$ \\ ${ }^{a}$ Kitakyushu National College of Technology, 5-20-1 Shii, Kokuraminami-ku, Kitakyushu-city, \\ Fukuoka 802-0985, Japan \\ bIsahaya Electronics Corporation, 6-41 Tsukuba-machi, Isahaya-city, Nagasaki 854-0065, Japan \\ ${ }^{c}$ Yamaguchi University, 2-16-1 Tokiwadai, Ube-city, Yamaguchi 755-8611, Japan \\ *Corresponding Author: kitazono@kct.ac.jp
}

\begin{abstract}
A luggage lifting occurs frequently, but clearance rate is only 16.9 percent. For the prevention of this problem, there are some systems that use radio intensity. However, the accuracy of these systems is not high, and this system cannot identify the luggage lifting and the luggage left behind. For the solution of the previously discussed problems, a new method to detect luggage lifting is proposed in this study. In the event of a luggage lifting, an owner is stopping and a lifting luggage is a walking state. By the use of acceleration sensors, the states of a luggage and the owner are able to be distinguished. In this method, the luggage lifting is detected based on the states. This method can identify the luggage lifting and the luggage left behind. In the event of a luggage lifting, the owner is notified with a sound, and the culprit is intimidated by warning sound. There is a strong likelihood that a criminal discard the luggage because the luggage warning sound is conspicuous. In addition, the accuracy to detect the luggage lifting is higher than that of conventional systems using radio intensity. By using this method, owners can be detected at an early stage luggage lifting. Therefore, the damage caused by the luggage lifting is reduced.
\end{abstract}

Keywords: luggage lifting, acceleration sensor, walking, luggage left behind.

\section{Introduction}

In the information society, people carry their smart phones and USB memories that store personally identifiable information are increasing ${ }^{(1,2)}$. Information leakage of personal information caused by the loss or theft of them has become a social problem ${ }^{(3,4)}$. A luggage lifting is one of the causes. The damage caused by the luggage lifting is not only information leakage, but also money damages. According to the Crime Situation in 2011 provided by the National Police Agency, the number of reported cases of the luggage lifting is $43,238^{(5)}$. However, clearance rate is only 16.9 percent. If the occurrence of the luggage lifting is reported to the owner, the damage caused by the luggage lifting is greatly reduced. In addition, by emitting a warning sound from the luggage, the damage caused by the luggage lifting is reduced more.

For the prevention of this problem, there are some systems that use radio intensity (Alarm away WSA600, Revex Co. Ltd. etc.). These systems estimate a distance between a luggage and the owner by radio intensity, and judges from the distance in between them that the luggage has been lost. However, the judgment is sometimes wrong, because radio intensity differs within an environment, even if the distance is the same ${ }^{(6)}$. For this reason, it may be too late to aware of the luggage lifting. When the owner has left the luggage, warning sound from the luggage may be annoying for people around the luggage because this system cannot identify the luggage lifting and the luggage left behind. On the other hand, the authors have developed the system for detect of luggage left behind ${ }^{(7,8)}$. This system can correctly be detect of a luggage has been left behind. However, recognition of luggage lifting is not considered in this system.

For the solution of the previously discussed problems, a new method to detect luggage lifting is proposed in this study. This method can identify the luggage lifting and the luggage left behind. In addition, the accuracy to detect the luggage lifting is higher than that of conventional systems 


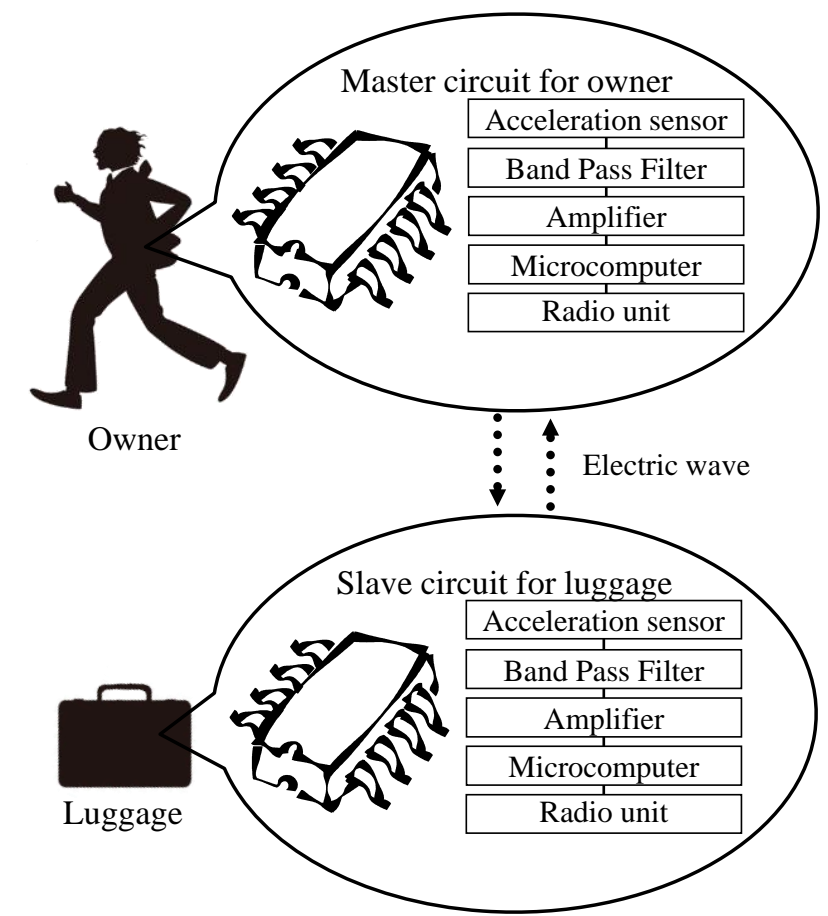

(a) Structure

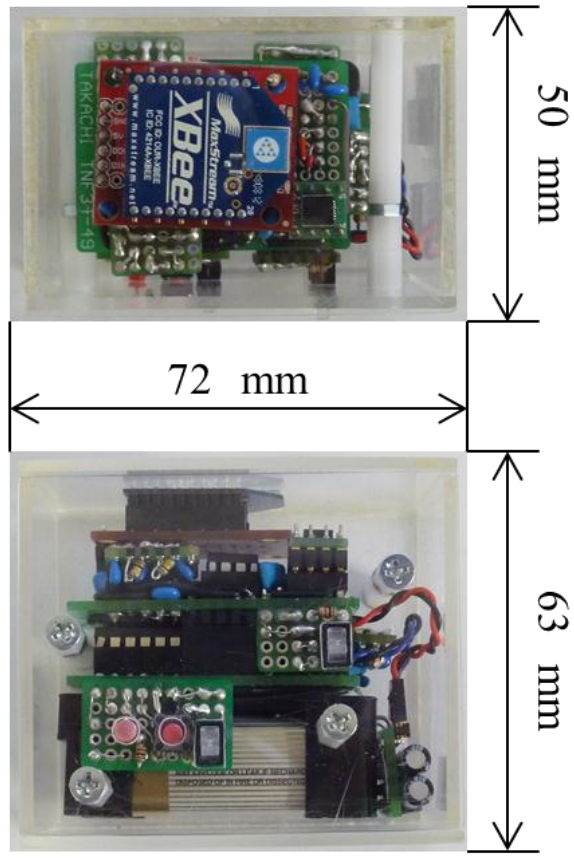

(b) Photo

Fig. 1. The proposed method to detect luggage lifting.

using radio intensity. By using this method, owners can be detected at an early stage luggage lifting. Therefore, the damage caused by the luggage lifting is reduced.

\section{Method to Detect Luggage Lifting}

\section{$2.1 \quad$ Structure}

Figure 1a shows the structure of this method to detect luggage lifting and Fig. 1b shows a photo of a circuit. Appearance of the master circuit and the slave circuit are the same. The slave circuit is attached to the luggage, and the owner has the master circuit. In the event of a luggage lifting, an owner is stopping and a lifting luggage is a walking state. In this method, a luggage lifting is recognized using the above properties.

Thus, we define the two states ("Walking" and "Stopping"). In the master circuit and the slave circuit, the two states are distinguished with acceleration sensors. The slave circuit transmits the state obtained by the sensor to the master circuit every 1 second. The radio unit (Xbee module, Digi International K. K.) is used for the communication of master and slave circuits. The master circuit detects a luggage lifting based on the states obtained by the two circuits, and the result is transmitted to the slave circuit. In

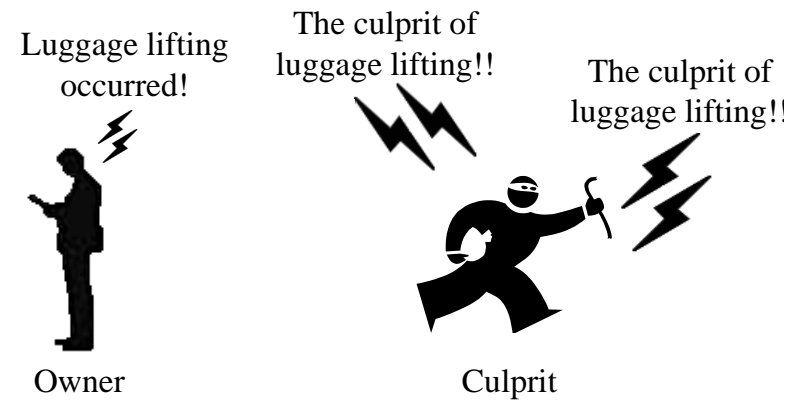

Fig. 2. The Appearance in the event of a luggage lifting.

the event of a luggage lifting as shown in Fig. 2, the master circuit notifies to the owner with a sound, and the slave circuit intimidates the culprit at warning sound. There is a strong likelihood that a criminal discard the luggage because the luggage warning sound is conspicuous.

\subsection{State Distinction}

Three axes acceleration sensors (Kionix Inc., KXM52-1050) are used for this state distinction. The acceleration sensors can detect acceleration on each axis. The fluctuation of acceleration by working (Frequency of about $0.1-30 \mathrm{~Hz}$ ) is extracted from the output of each axis 


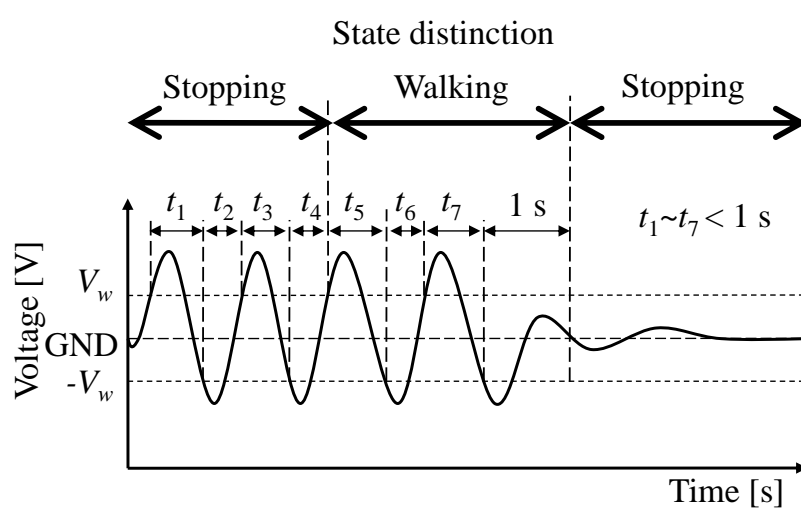

Fig. 3. The output of the acceleration sensor when a person is walking.

of the sensors. It is then sent to the microcomputer. The microcomputer detects the state by every 0.1 second. The state is then classified into one of the two states ("Walking" and "Stopping").

(a) Walking

The output of the acceleration sensor and the change of state when a person is walking are shown in Fig. 3. In Fig. 3 , a threshold value of $V_{w}$ is defined. If the time $t_{1}$ as shown in Fig. 3 is shorter than 1 second, it is judged as the start of walking. If these are repeated within every 1 second, it is regarded as walking. In this study, we defined the time after three steps as the start of "Walking". The above determination is performed for each output of the acceleration sensor. If there is a "Walking" in to any of the results, Its state is determined during "Walking".

(b) Stopping

The states other than "Walking" are judged "Stopping".

\subsection{Detection of Luggage Lifting}

The master circuit detects luggage lifting by using the state ("Walking" and "Stopping") of the past 10 times every 1 second. In the event of a luggage lifting, an owner is "Stopping" and a lifting luggage is "Walking". In this situation, the master circuit judges "Stopping" and the slave circuit judges "Walking". When this state continues more than 5 times, it is regarded as the luggage lifting. The other cases are not regarded as luggage lifting.

\section{Experiments}

\subsection{Decision of Threshold Voltage}

Threshold voltage $\left(V_{w}\right)$ for the state distinction was decided in this experiment. The output of the acceleration

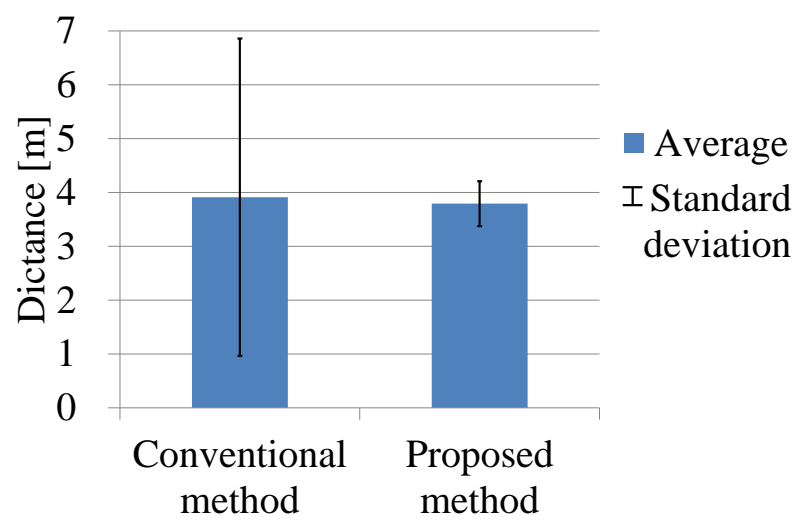

Fig. 4. The distance at the detection of a luggage lifting.

sensors were measured at the following conditions of a person stopped and walking slowly $(3 \mathrm{~km} / \mathrm{h}$ ), normally (4 $\mathrm{km} / \mathrm{h})$, and quickly $(6 \mathrm{~km} / \mathrm{h})$. Six people performed this experiment. Each person tried the experiment 10 times. As a result, a recognition rate of 100 percent for "Walking" were obtained within the range of $0.5 \sim 0.9 \mathrm{~V}$ of $V_{w}$. Therefore, $V_{w}$ was decided as $0.6 \mathrm{~V}$.

In all the following experiments, the master circuit was attached to the owner and the slave circuit was attached to the luggage.

\subsection{In the Case of Luggage Lifting}

In this experiment, the detection by the proposed method was examined in the event of a luggage lifting. In such a situation, the owner is stopping, and the luggage is walking away from the owner. The experiment was carried out at four places; three different rooms and an outdoor location. Six people were tried and they walked at speeds of slowly (3 km/h), normally (4 km/h), and quickly $(6 \mathrm{~km} / \mathrm{h})$. Each person tried the experiment 5 times.

The conventional method using radio intensity was also tried for reference.

The recognition rate of a luggage lifting was 100 percent in proposed method. The distance at the detection of a luggage lifting is shown in Fig. 4. The average distance at each speed and the standard deviation are shown in the figure. From the Fig. 4, it is understood that the average distance of the proposed method was almost the same as that of the conventional method, but the standard deviation was smaller than that of the conventional method. Thus, the accuracy of the detection by the proposed method was higher than that of the conventional method. In addition, in the event of a luggage lifting, it was confirmed that the master circuit sounded and the slave circuit warned. 


\subsection{In the Case of No Luggage Lifting}

In this experiment, the detection by the proposed method was examined when there was no luggage lifting. When an owner possesses a luggage such as baggage, there are two situations:

(a) The owner is stopping with the luggage.

(b) The owner is walking with the luggage.

Ten people performed this experiment. As a result, there was no false detection for any of the situations.

\section{Conclusions}

A new method to detect luggage lifting was proposed in this study. By the use of acceleration sensors, the states of a luggage and the owner are able to be distinguished. As the result, it can correctly be detect a luggage lifting. In the event of a luggage lifting, the owner is notified with a sound, and the culprit is intimidated by warning sound. There is a strong likelihood that a criminal discard the luggage because the luggage warning sound is conspicuous.

In this study, we proposed a method to detect only the luggage lifting. As future prospects, we are planning to develop a system to detect both the luggage lifting and the luggage left behind.

\section{References}

(1) "Monthly Consumer Confidence Survey covering all of Japan", Cabinet Office, Government of Japan, 2012

(2) "Research Report of Malware that Infects via USB Memory", Japan Computer Emergency Response Team Coordination Center, p. 1, 2009

(3) "Survey Report on the 2011 Information Security Indentation", NPO Japan Network Security Association, p. 13, 2012

(4) Kiyoto Onodera : "Information Leakage and Countermeasure of It - The Pitfalls with Countermeasure of Spyware -", Info-Future, No. 21, pp. 22-23, 2005

(5) "Crime Situation in 2011", National Police Agency, p. 103, 2011

(6) Yoshikazu Ohta, Masashi Sugano, and Masayuki Murata : "A Data Collecting Technique for Localization in Wireless Sensor Networks", IEICE Technical Report, Information Networks, Vol. 102, No. 437, pp. 31-36, 2004

(7) Yuhki Kitazono, Takeshi Inoue, Makoto Miyauchi, and Seiichi Serikawa : "Development of System for
Prevention of Thing Left Behind Using Acceleration Sensors and RFID Tag", IEEJ Transactions on Sensors and Micromachines, Vol. 128, No. 9, pp. 364-365, 2008

(8) Yuhki Kitazono, Yuji Ohta, Shota Nakashima, Makoto Miyauchi, and Seiichi Serikawa : "Proposal of system for detection of thing left behind", Procedia - Social and Behavioral Sciences, Vol. 2, Iss. 1, pp. 186-192, 2010 